\title{
Universal antibiotic tolerance arising from antibiotic-triggered accumulation of redox metabolites
}

Kui Zhu, ${ }^{1,2}$ Shang Chen, ${ }^{1}$ Tatyana A. Sysoeva, ${ }^{2}$ Lingchong You $2,3,4+$

${ }^{1}$ Beijing Advanced Innovation Center for Food Nutrition and Human Health, College of Veterinary Medicine, China Agricultural University, Haidian, Beijing 100193, China

2 Department of Biomedical Engineering, Duke University, Durham, North Carolina, 27708, USA

${ }^{3}$ Center for Genomic and Computational Biology, Duke University, Durham, North Carolina, 27708, USA

${ }^{4}$ Department of Molecular Genetics and Microbiology, Duke University School of Medicine, North Carolina, 27710, USA

${ }^{+}$Correspondence and requests for materials should be addressed to Lingchong You.

E-mail: you@duke.edu; Tel: 919-660-8408; Fax: 919-668-0795 


\section{Abstract}

Pseudomonas aeruginosa is an opportunistic pathogen that often infects open wounds or patients with cystic fibrosis. Once established, P. aeruginosa infections are notoriously difficult to eradicate. This difficulty is in part due to the ability of $P$. aeruginosa to tolerate antibiotic treatment at the individual-cell level or through collective behaviors. Here we describe a new mechanism by which $P$. aeruginosa tolerates antibiotic treatment by modulating its global cellular metabolism. In particular, treatment of $P$. aeruginosa with sublethal concentrations of antibiotics covering all major classes promoted accumulation of the redox-sensitive phenazine - pyocyanin (PYO). PYO in turn conferred general tolerance against diverse antibiotics for both $P$. aeruginosa and other Gram-negative and Gram-positive bacteria. We show that PYO promotes energy generation to enhance the activity of efflux pumps, leading to enhanced antibiotic tolerance. This property is shared by other redox-active phenazines produced by $P$. aeruginosa. Our discovery sheds new insights into the physiological functions of phenazines and has implications for designing effective antibiotic treatment protocols.

\section{Author Summary}

Antibiotic tolerance can facilitate the evolution of resistance, and here we describe a previously unknown mechanism of collective antibiotic tolerance in Pseudomonas aeruginosa. In particular, $P$. aeruginosa treated with sublethal concentrations of antibiotics covering all major classes promotes accumulation of pyocyanin (PYO), an important virulence factor. In turn, PYO confers general tolerance against diverse antibiotics for both $P$. aeruginosa and other bacteria. Our discovery is a perfect example of what Nietzsche once said: That which does not kill me makes me stronger. 


\section{Introduction}

The overuse and misuse of antibiotics have led to a global crisis (1): bacteria have developed resistance against every existing antibiotic and are doing so at an alarming rate, considering the timescale at which new antibiotics progress from development to clinical application $(2,3)$. The drying antibiotic pipeline further heightens the global threat created by infectious bacteria $(4,5)$. A critical approach to the problem is developing ways to revitalize existing antibiotics (6). To extend the use of existing antibiotics, we need to develop a mechanistic understanding of the diverse ways by which bacteria survive antibiotics. Such an understanding is critical for designing therapeutic approaches that subvert the survival tactics by bacterial pathogens.

Past efforts on antibiotic resistance have focused on responses of individual cells, such as mutations in the antibiotic targets, enzymatic activity that inactivates antibiotics, and increased activation of efflux pumps (6). Unlike antibiotic resistance, which is due to inherited or acquired mutations $(7,8)$, tolerance reflects the ability of individual cells (9) or cell populations (10) to survive antibiotic treatment without acquiring new mutations. However, it has been long realized that antibiotic tolerance precedes resistance (11-13). Recent in vitro experiments have shown that antibiotic tolerance can facilitate the evolution of resistance (14). In particular, antibiotic tolerance can pave the way for the rapid subsequent emergence of antibiotic resistance in bacteria; thus, preventing the evolution of tolerance may shed light on alternative strategies for antibiotic treatments. Importantly, the ability to survive antibiotic treatment is typically considered an intrinsic property of the single bacterial cells or bacterial populations, before antibiotics are applied. In contrast to these prevailing views, here we describe a previously unknown mechanism of collective antibiotic tolerance in Pseudomonas aeruginosa. 
PYO, one of the most studied phenazines, is a redox-active metabolite giving the characteristic blue-green pigment of $P$. aeruginosa cultures (15). PYO is typically produced when $P$. aeruginosa enters the stationary phase when the cell density is high (16). Recent studies have demonstrated a number of physiological roles of PYO, such as serving as a signaling compound (17), facilitating biofilm development (18), promoting iron acquisition (19), and influencing colony formation (20). It is also known to confer a broad-spectrum antibiotic activity (21). Our work provides novel insights into the biological function of bacterial redox-active metabolites, and suggests a role of such metabolites, including PYO and other phenazines, in the survival of bacteria during acute stress.

\section{Results}

\section{Subinhibitory concentrations of antibiotics induce PYO accumulation}

We observed that sub-inhibitory concentrations of kanamycin (Kan), a commonly used aminoglycoside antibiotic, induced a blue-greenish color change in P. aeruginosa PAO1 (ATCC 47085) cultures. PYO is a typical pigment for such color change (15). We confirmed this notion by purifying PYO using an established method (22), which also allowed us to measure PYO accumulation. The induction of PYO followed a biphasic dependence on the Kan dose. The blue-green color of the cultures became more visible with increasing Kan concentrations (Fig.

1A). This color change is consistent with direct quantification of PYO in the supernatants (Fig.

1B). Concurrently, the bacterial density (A600 nm) decreased with the Kan concentrations (Fig.

1B). This enhanced PYO accumulation related to the culture without antibiotic treatment was transient, as evident in the direct measurements of PYO purified from the supernatants of cultures treated with $20 \mu \mathrm{g} / \mathrm{mL}$ Kan (Fig. 1C). 
We wondered whether the enhanced PYO accumulation could represent a general stress response to antibiotic treatment. To test this notion, we measured PAO1 strain responses to other antibiotics with different modes of action, including chloramphenicol that targets ribosome 50 S subunit, norfloxacin (quinolone family) that inhibits DNA replication, polymyxin B (polypeptide family) that alters cell membrane permeability, and carbenicillin ( $\beta$-lactams) that inhibits cell-wall synthesis. All tested antibiotics promoted accumulation of PYO in a similar biphasic manner (Supplementary, Fig. S1A). This response was not unique to PAO1. The PA14 strain, which is known to produce more PYO than PAO1 (17), exhibited qualitatively the same responses to these antibiotics (Supplementary, Fig. S1B). The biphasic PYO accumulation and its temporal dynamics reconcile the apparently contradictory conclusions on PYO accumulation under antibiotic stresses measured previously by single-point measurements (23-25).

Cyclic di-GMP (c-di-GMP), an intracellular second messenger, has been shown to confer tolerance to antibiotics $(26,27)$. Moreover, c-di-GMP has been shown to promote PYO production (28). These studies suggest a potential role of c-di-GMP in antibiotic-mediated PYO accumulation. Consistent with this notion, we observed increased levels of c-di-GMP in PAO1 in response to several antibiotics (Fig. 1D). In the absence of antibiotics, the accumulation of c-di-GMP was approximately 2.5 fold higher in PA14 than in PAO1 during early stationary phase (Supplementary, Fig. S2A). This observation provides a potential explanation for the typically higher PYO accumulation in PA14 than in PAO1. Additionally, exogenous GTP - the precursor of c-di-GMP (27), leads to increased c-di-GMP over time and increased PYO 
accumulation in a dose-dependent manner (Supplementary, Fig. S2B, S2C, and S2D). This result confirms the recent finding that PYO production is c-di-GMP dependent (29).

\section{Accumulated PYO enhances antibiotic tolerance in bacteria}

We wondered whether the enhanced accumulation of PYO could represent a survival mechanism for the population under acute antibiotic stress. Indeed, growth of $P$. aeruginosa PAO1 in the presence of Kan was enhanced by exogenously added PYO (Fig. 2A). This PYO-mediated tolerance was effective against other aminoglycosides (gentamicin, streptomycin and tobramycin) and antibiotics of other classes (norfloxacin, chloramphenicol and carbenicillin)

(Fig. 2A). In the presence of PYO, PAO1 cultures exhibited a much shorter lag time before recovering in the presence of an antibiotic, in comparison to cultures without exogenously added PYO (Supplementary, Fig. S3). Endogenous PYO, if accumulated at a sufficiently high level such as in the cystic fibrosis respiratory tract (30), can also provide protection. In addition, PYO-mediated tolerance was maintained when PAO1 was cultured in other media or different oxygen conditions (Supplementary, Fig. S4). An exception to the general PYO-mediated tolerance was polymyxin B, where PYO enhanced its ability to inhibit bacterial growth (Fig. $2 \boldsymbol{A})$.

\section{Oxidized PYO serves as electron acceptors to mediate antibiotic tolerance}

As the PYO-mediated tolerance was general against different classes of antibiotics for diverse bacteria, it likely resulted from PYO-mediated modulation of cellular physiology that is common for all these bacteria. As a redox-active molecule (33), PYO can exist in reduced or oxidized state, depending on its chemical environment. The oxidized form of PYO can act as an electron acceptor to modulate the global energy metabolism in the cell by regulating the flux of electron shuttling (34). Similar functions have been proposed for other chemicals that can act as 
electron acceptors. For instance, the electron-shuttling ability of oxidized tetrathionate (35) and nitrate (36) has been implicated in enhancing bacterial growth advantage by promoting respiration. Additionally, hydrogen sulfide has been shown to enhance bacterial survival during antibiotic stress (37). Consistent with this notion, several reductants, including nicotinamide adenine dinucleotide (NADH), $\beta$-mercaptoethanol, and $N$-acetyl- $L$-cysteine (NAC), all eliminated PYO-mediated antibiotic tolerance (Fig. $3 \boldsymbol{A}$ and Supplementary, S6A). By themselves, these chemicals did not affect $P$. aeruginosa growth or response to antibiotics. The presence of these reductants in excess prevented significant accumulation of the oxidized PYO, thus interfering with its ability to act as an electron acceptor. As a side, our results might provide an explanation to previously observed unknown benefit of reductants as antibiotic-adjuvants in treating CF patients (38), and suggest new strategy to devise antibiotic adjuvants.

If electron-shuttling ability is critical for PYO-mediated antibiotic tolerance, we reasoned that other redox-active phenazines might exhibit similar protective effects. Indeed, several natural analogs of PYO, including phenazine-1-carboxylic acid (PCA), phenazine-1-carboxamide (PCN) and 1-hydroxyphenazine (1-OHPHZ), promoted P. aeruginosa survival during antibiotic exposure (Supplementary, Fig. S6B). Further confirming this notion, two other well-established electron acceptors, 2,6-anthraquinone disulfonate (AQDS) (39), and methylene blue (MB) (40), also conferred antibiotic tolerance in $P$. aeruginosa (Fig. $3 \boldsymbol{B}, \quad 3 \boldsymbol{C}$ and

\section{Supplementary, S6B).}

\section{Oxidized PYO stimulates energy metabolism to pump antibiotics out}

Studies have suggested a role of PYO in promoting ATP production (34), particularly when 
cells enter stationary phase or are under stress (40). Consistent with this notion, we observed that exogenously added PYO promoted ATP accumulation during stationary phase (Supplementary, Fig. S7). An increase in ATP accumulation can facilitate generation of the proton-motive force (PMF) (41), which plays a critical role in promoting bacterial survival (but not growth) under anaerobic condition (34), in cell division (42) and increased efficiency of efflux pumps (EPs) (43, 44). To test this notion, we measured the membrane potential with the carbocyanine dye $\mathrm{DiOC}_{2}(3)$, which confirmed an elevated PMF in the presence of PYO (Fig. 3D). Additionally, the multidrug resistance phenotype in $P$. aeruginosa is in major part due to the expression of abundant EP systems $(45,46)$. For example, the MexAB-OprM pump plays a key role in the intrinsic resistance for several types of antibiotics (44). An increasing efficiency of EPs, mediated by an elevated PMF, would provide an explanation for the general PYO-mediated tolerance to a wide variety of antibiotics. The PYO-mediated promotion of PMF also explains the lack of PYO-mediated tolerance against polymyxin B (Fig. $2 \boldsymbol{A}$ and Supplementary, S3B): the loss of cell membrane integrity caused by polymyxin B completely eliminates the PMF. This is consistent with the previous observation that none of EPs extrude polymyxin B in $P$. aeruginosa (47), because EPs are anchored in membrane and most are driven by PMF (43-45). Aside from polymyxin B, an increased PMF may not always benefit the cells. In particular, as the uptake of aminoglycosides is energy dependent, an increased PMF can enhance their uptake and subsequent inhibition of bacterial growth (48) (i.e. by overcoming the increased efficiency of EPs). Thus, we reasoned that PYO-mediated tolerance would be eliminated at high levels of aminoglycosides. Indeed, $100 \mu \mathrm{g} / \mathrm{mL}$ Kan and $10 \mu \mathrm{g} / \mathrm{mL}$ gentamycin effectively suppressed $P$. aeruginosa growth, further demonstrating that PYO mediated tolerance was due to PMF. 
Given this model (Fig. 3C), we expected that inhibition of the hydrolysis of ATP would abolish PYO-mediated antibiotic tolerance. Indeed, sodium azide, which inhibits the $\mathrm{F}_{1}$ subunit of ATP synthase (49), abolished the tolerance (Fig. 3E). Similarly, N,N'-dicyclohexylcarbodiimide (DCCD), which blocks the proton translocation channel in the $\mathrm{F}_{0}$ subunit of ATP synthase (34), also reduced the PYO-mediated tolerance (Supplementary, Fig. S8A). Further confirming the role of PMF, ionopore carbonyl cyanide $m$-chlorophenyl hydrazone (CCCP), which directly dissipates PMF, also reduced PYO-mediated tolerance (Fig. $\mathbf{3 E}$. Furthermore, both CССР and sodium azide enhanced growth inhibition by antibiotics, even in the presence of PYO (Supplementary, Fig. S8B and S8C).

We further tested modulation of EP efficiency by quantifying intracellular accumulation of ethidium bromide (EtBr), a well-established substrate of EPs in P. aeruginosa (44). Indeed, PYO accelerated the extrusion of EtBr by cells (Fig. $4 \boldsymbol{A}$ ). If an enhanced efficiency of EPs was responsible for PYO-mediated antibiotic tolerance, we would expect chemical inhibitors of EPs to abolish this tolerance. We tested this notion by using phenylalanine-arginine $\beta$-naphthylamide $(\mathrm{PA} \beta \mathrm{N})$, which inhibits the resistance nodulation cell division (RND) pumps, the major contributors to antibiotic resistance in P. aeruginosa (50). Indeed, PA $\beta \mathrm{N}$ reduced PYO-mediated tolerance in a dose-dependent manner (Supplementary, Fig. S8D).

When the EPs are already expressed at a sufficiently high level, an elevated pump activity in the presence of PYO was primarily due to an enhanced energetic state by the cells, instead of up-regulation of pump genes. For example, various pump gene expression was not regulated (Supplementary, Fig. S9). P. aeruginosa features a transcriptional factor, SoxR, which is activated by phenazines (20). These results also indicate that the PYO-mediated protection is not due to its 
regulation of SoxR (Supplementary, Fig. S10A, which can modulate expression of EPs (17). The tolerance was not mediated by SoxR in P. aeruginosa, however, this result does not exclude its potential role in other bacteria such as E. coli. Moreover, PAO-JP2 strain has multiple quorum sensing (QS) genes knocked out (31), combining the assay with the addition of exogenous QS signals (Supplementary, Fig. S10B), and suggesting a dispensable role of QS in PYO-mediated tolerance. Altogether, we found that the PYO mediated tolerance was neither through working as a signal to regulate the hierarchical QS network (Supplementary, Fig. S6), nor through activating SoxR.

\section{Discussion}

The antibiotic-induced early PYO accumulation could result from reduced degradation or increased production of PYO. For example, antibiotics could inhibit enzymes involved in PYO degradation, though such enzymes have not yet been identified in P. aeruginosa (51). Alternatively, cells could increase PYO synthesis to counteract stress, akin to that experienced by cells entering the stationary phase. It has been well established that different antibiotics can trigger accumulation of reactive oxygen species (ROS) (52). Likewise, quorum sensing (QS) has been implicated in PYO metabolism (17). However, our experiments demonstrated that the antibiotic-mediated PYO accumulation was unlikely due to these mechanisms (Supplementary, Fig. S11).

Collectively, our results reveal a previously unknown mechanism by which $P$. aeruginosa can develop collective antibiotic tolerance (10) mediated by PYO. Both the induction of PYO and the PYO-mediated tolerance are general: all tested antibiotics promoted accumulation of PYO; and PYO conferred tolerance against all antibiotics with the exception of polymyxin B (Fig. $4 \boldsymbol{B})$. 
Moreover, the PYO-mediated protection is not limited to the producing cells; rather, PYO also enhances survival of other P. aeruginosa strains and other bacteria. This property highlights a particular challenge in treating infections involving $P$. aeruginosa: incomplete suppression of $P$. aeruginosa by antibiotics would enhance growth of survivors, as well as other pathogens in the growth environment. Nevertheless, the PYO-mediated tolerance results from the cell's intrinsic ability to counteract a disruption in the cellular state, whereas PYO itself can be considered as a stress. As such, this tolerance is a double-edged sword. An elevated energy generation can enhance efficacy of certain antibiotics by promoting their uptake (48). Also, if pushed beyond cell's buffering capacity, the PYO-mediated disruption in the redox balance can inhibit growth $(15,20)$. Thus, this tolerance mechanism also presents an opportunity for designing treatment strategies that can synergistically enhance effects of antibiotics. For instance, specific inhibitors can be developed to target the multiple enzymes involved for the synthesis of phenazines, antioxidants can be used as adjuvants to counteract the protective effect of PYO.

\section{EXPERIMENTAL SECTION}

Chemicals and strains Antibiotics used in this study include carbenicillin, chloramphenicol, gentamicin, kanamycin, norfloxacin, polymyxin B, streptomycin and tobramycin. Oxidized forms of pyocyanin (PYO) and other phenazines were prepared as previously described (1). Beta-mercaptoethanol, nicotinamide adenine dinucleotide (NADH) and $N$-acetyl- $L$-cysteine (NAC) were used to reduce oxidized form of PYO to its reduced form. 2,6-anthraquinone disulfonate (AQDS) and methylene blue (MB) were used as electron acceptors. Carbonyl cyanide $m$-chlorophenyl hydrazone (CCCP) was used to dissipate the proton motive force. N,N'-dicyclohexylcarbodiimide (DCCD) and sodium azide were used to inhibit different subunits of ATP synthase. Ethidium bromide (EtBr) was used to test the function of efflux pumps, and phenylalanine-arginine $\beta$-naphthylamide (PA $\beta N)$ was used to inhibit the efficiency of efflux pumps. N-butyryl-L-homoserine lactone (C4-HSL) and 
N-3-oxo-dodecanoyl-L-homoserine lactone (C12-HSL) were used as quorum sensing molecules. All chemicals were purchased from Sigma-Aldrich and Cayman Chemical.

P. aeruginosa, Escherichia coli, Salmonella typhimurium, Bacillus cereus, Bacillus subtilis and Staphylococcus aureus strains were used in this study. More details of these strains were shown in Supporting

\section{Information Table S1.}

PYO accumulation in culture tubes Different concentrations of antibiotics were added to 4 $\mathrm{mL}$ Luria-Bertani $(\mathrm{LB})$ broth $(5 \mathrm{~g} / \mathrm{L}$ yeast extract, $10 \mathrm{~g} / \mathrm{L}$ tryptone and $10 \mathrm{~g} / \mathrm{L} \mathrm{NaCl}$, Genesee Scientific) in culture tubes (Genesee Scientific) for P. aeruginosa PAO1 and PA14 strains. Cultures at exponential phase were adjusted to $\mathrm{A} 600 \mathrm{~nm}=0.2$, subsequently $10 \mu \mathrm{L}$ cultures were resuspended in $4 \mathrm{~mL}$ fresh $\mathrm{LB}$ medium. All the tubes were cultured in a shaker (New Brunswick Scientific) at $37^{\circ} \mathrm{C}, 250 \mathrm{rpm}$. When appropriate, the photos of cultured tubes treated with various antibiotics were taken and cell density (A600 nm) were measured at $600 \mathrm{~nm}$. Excreted PYO was quantified based on the presence of pink to deep red color in acidic solution as described previously (2). Briefly, $2.5 \mathrm{~mL}$ supernatant from LB broth was mixed with $1.5 \mathrm{~mL}$ chloroform at specified time points. PYO in the chloroform phase was then extracted into 0.5 $\mathrm{mL} 0.2 \mathrm{~mol} / \mathrm{L}$ hydrochloric acid $(\mathrm{HCl})$. After centrifugation, the absorbance of the top layer was measured at $520 \mathrm{~nm}$. Concentrations $(\mu \mathrm{g} / \mathrm{mL})$ of PYO were expressed as micrograms of PYO per milliliter supernatant, determining by multiplying the absorbance at $520 \mathrm{~nm}$ (A520 nm) by 17.072 .

Growth condition in multi-well plates For routine growth, bacterial strains were all cultured in $100 \mu \mathrm{L} \mathrm{LB}$ medium in 96-well cell culture plates (Costar, Corning) for long-term measurement with a plate reader (Victor, Perkin Elmer). Exponential growth cultures of bacteria were adjusted to $\mathrm{A} 600 \mathrm{~nm}=0.2$, then $1: 20$ diluted in $100 \mu \mathrm{L}$ fresh $\mathrm{LB}$ broth containing various concentrations of antibiotics either in the presence or absence of exogenous $2 \mu \mathrm{g} / \mathrm{mL}$ oxidized PYO or other phenazines. P. aeruginosa PAO1 was also cultured in other media, including brain-heart infusion (BHI), M9 and 2xYT media. For growth dynamic measurements, $50 \mu \mathrm{L}$ mineral oil (Sigma) was added into each well to prevent evaporation. The absorbance was measured by an Infinite 200 Pro plate reader (Tecan) at the wavelength of $600 \mathrm{~nm}$ (A600). Furthermore, oxygen permeable sealing membrane (Diversified Biotech) was used to determine whether increasing oxygen concentration could increase antibiotic tolerance. Additionally, LB medium supplemented with 
different reducing agents $(100 \mu \mathrm{mol} / \mathrm{L}$ beta-mercaptoethanol, $15 \mu \mathrm{mol} / \mathrm{L} \mathrm{NADH}$ and 13 mmol/L NAC) were simultaneously added with the oxidized form of PYO.

c-di-GMP assay $P$. aeruginosa PAO1 was cultivated in LB at $37^{\circ} \mathrm{C}$ for $8 \mathrm{~h}$ with shaking. $10 \mathrm{~mL}$ of cultures $(\mathrm{OD} 600=1.2)$ in the presence or absence of antibiotics at room temperature for 3 hrs, were harvested by quick centrifugation $(10000 \mathrm{~g})$ at $4{ }^{\circ} \mathrm{C}$ for $10 \mathrm{~min}$. Cells were washed by 2 $\mathrm{mL}$ of water and collected by quick centrifugation $(10000 \mathrm{~g})$ at $4{ }^{\circ} \mathrm{C}$ for another $10 \mathrm{~min}$. Cell

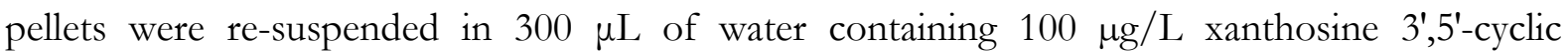
monophosphate (cXMP, Sigma) as the internal standard and incubated at $4{ }^{\circ} \mathrm{C}$ for $15 \mathrm{~min}$, then heating at $95{ }^{\circ} \mathrm{C}$ for $10 \mathrm{~min}$. After cooling at room temperature for $10 \mathrm{~min}$, the suspension was centrifuged $(11000 \mathrm{~g})$ at $4{ }^{\circ} \mathrm{C}$ for another $15 \mathrm{~min}$. Cell pellets were extracted with $200 \mu \mathrm{L}$ of $65 \%$ ice-cold ethanol at $4{ }^{\circ} \mathrm{C}$ for twice. The supernatants were evaporated until dryness at $40{ }^{\circ} \mathrm{C}$ under nitrogen gas. The residues were re-suspended in $200 \mu \mathrm{L}$ of $0.5 \mathrm{mmol} / \mathrm{L}$ ammonium acetate containing $0.3 \%(\mathrm{v} / \mathrm{v})$ acetic acid for subsequent UPLC-MS/MS analysis. Briefly, eluent A consisted of $0.5 \mathrm{mmol} / \mathrm{L}$ ammonium acetate containing $0.3 \%(\mathrm{v} / \mathrm{v})$ acetic acid and eluent B was methanol. The injection volume was $10 \mu \mathrm{L}$ and the flow rate was $0.3 \mathrm{~mL} / \mathrm{min}$. Temperature for BEH Shield RP 18 column (Waters) was set at $30{ }^{\circ} \mathrm{C}$. The concentration of c-di-GMP was quantified by Xevo TQ triple quadrupole mass spectrometer (Waters) equipped with an electro spray ionization (ESI) source for multiple reaction monitoring (MRM) analysis in positive ionization mode. The following MRM transitions were detected for the quantification of c-di-GMP at $\mathrm{m} / \mathrm{z}$ of $+691 / 152$ (quantifier), and confirmatory signals were monitored $\mathrm{at} \mathrm{m} / \mathrm{z}$ of $+691 / 135$ and $+691 / 248$ (qualifier). Final concentration of c-di-GMP was expressed as the ratio of c-di-GMP (ng) to bacterial protein (mg). Protein content was tested by bicinchoninic acid (BCA) assay (Beyotime). Briefly, $2 \mathrm{~mL}$ bacterial culture was harvested at $10000 \mathrm{~g}$ and washed by $2 \mathrm{~mL}$ of water, then collected by quick centrifugation $(10000 \mathrm{~g})$ at $4{ }^{\circ} \mathrm{C}$ for another $10 \mathrm{~min}$. Subsequently, the cell pellet was dissolved in $800 \mu \mathrm{L}$ of $0.1 \mathrm{~mol} / \mathrm{L}$ sodium hydroxide and heated at $95{ }^{\circ} \mathrm{C}$ for $15 \mathrm{~min}$ before testing. The levels of c-di-GMP in $P$. aeruginosa under antibiotic treatments were normalized with that without antibiotics.

ATP assay The ATP contents of $P$. aeruginosa PAO1 in LB were measured by the Enhanced ATP Assay Kit (Beyotime, Shanghai, China) according to the manufacture's instruction. Briefly, $5 \mathrm{~mL}$ of early stationary-phase bacteria $(\mathrm{A} 600 \mathrm{~nm}=0.7)$ covered by $1 \mathrm{~mL}$ mineral oil in cultural 
tubes were spiked with $2.5 \mu \mathrm{g} / \mathrm{mL}$ PYO at different time points at room temperature. $4 \mathrm{~mL}$ bacterial culture was harvested by centrifugation $(10000 \mathrm{~g})$ at $4{ }^{\circ} \mathrm{C}$ for $10 \mathrm{~min}$, then the cell pellets were lysed by $200 \mu \mathrm{L}$ lysing buffer. Subsequently, the supernatant was obtained by centrifugation $(12000 \mathrm{~g})$ at $4{ }^{\circ} \mathrm{C}$ for $5 \mathrm{~min}$. Lastly, the bioluminescence signals were determined by SpectraMax ${ }^{\circledR}$ M5 Microplate Reader (Molecular Devices LLC), based on luciferase catalyzes the formation of light from ATP and luciferin.

Membrane potential measurement The membrane potential of $P$. aeruginosa PAO1 was measured by the BacLight ${ }^{\text {TM }}$ Bacterial Membrane Potential Kit (B34950, Molecular Probes). Cultures at exponential phase were added to fresh LB medium (approx. $10^{6} \mathrm{CFU}$ ) and 100 $\mu \mathrm{mol} / \mathrm{L}$ 3,3'-diethyloxacarbocyanine iodide $\left(\operatorname{DiOC}_{2}(3)\right.$, membrane stain) with $20 \mu \mathrm{g} / \mathrm{mL}$ streptomycin in a 96-well black plate (Costar, Corning), either in the presence or absence of 2 $\mu \mathrm{g} / \mathrm{mL}$ PYO and/or $10 \mu \mathrm{mol} / \mathrm{L}$ CCCP, or both. Subsequently, $50 \mu \mathrm{L}$ mineral oil was added into each well and the plate was analyzed on an Infinite 200 Pro plate reader (Tecan). The red/green fluorescent intensities for each well were collected with the excitation wavelength at $488 \mathrm{~nm}$ and with the emission wavelengths at $525 \mathrm{~nm}$ and $590 \mathrm{~nm}$, respectively. The normalized fluorescent values were used to determine the relative changes of proton motive force (PMF).

Accumulation and extrusion assays The accumulation of ethidium bromide (EtBr) was carried out in a 96-well black plate (Costar, Corning). Cultures of P. aeruginosa PAO1 at exponential phase $(\mathrm{A} 600 \mathrm{~nm}=0.2)$ were 1:20 diluted into $100 \mu \mathrm{L}$ fresh LB medium, supplemented with $2.5 \mu \mathrm{g} / \mathrm{mL}$ EtBr. $2 \mu \mathrm{g} / \mathrm{mL}$ PYO and $20 \mu \mathrm{g} / \mathrm{mL}$ streptomycin were simultaneously added in the presence or absence of $10 \mu \mathrm{mol} / \mathrm{L} \mathrm{CCCP.} \mathrm{For} \mathrm{the} \mathrm{extrusion} \mathrm{of} \mathrm{EtBr}$ assay, P. aeruginosa PAO1 culture was pre-treated with $50 \mu \mathrm{g} / \mathrm{mL}$ streptomycin in the presence of $2.5 \mu \mathrm{g} / \mathrm{mL}$ EtBr for $30 \mathrm{~min}$ to allow accumulation of EtBr. The bacteria were centrifuged at 8 $000 \mathrm{~g}$ for $3 \mathrm{~min}$ to remove the culture media, and washed by phosphate-buffered saline (PBS, Sigma) twice. The bacterial pellets were replaced by LB broth in the presence or absence of PYO. Lastly, $50 \mu \mathrm{L}$ mineral oil was added into each well and the plate was measured for $10 \mathrm{~h}$. The absorbance (A600 nm) and fluorescence of the conjugate of EtBr binding to nucleic acid were recorded with an Infinite 200 Pro plate reader (Tecan). The excitation wavelength was set at 488 $\mathrm{nm}$ and the emission wavelength was at $590 \mathrm{~nm}$.

qRT-PCR analysis The primers used for the quantification of efflux pump gene expression are 
listed in SI Appendix. Table S2. Total RNA was isolated from P. aeruginosa cells grown in the presence or absence of streptomycin and PYO with conditions similar to the above. Shortly, overnight cell cultures were 1 to 100 folds diluted into fresh LB medium (pH 7.0) and distributed into a 6-well plate. PYO $(2 \mu \mathrm{g} / \mathrm{mL})$ or/and streptomycin $(20 \mu \mathrm{g} / \mathrm{mL})$ were added and incubated at $30^{\circ} \mathrm{C}$ for 2, 5, $10 \mathrm{hrs}$ before the cells were collected for analyses. RNA was extracted using the Qiagen RNAeasy kit (Qiagen) followed by DNase I digestion. cDNA was obtained by reverse transcription of the total RNA using the Applied Biosystems high-capacity cDNA Reverse Transcription kit (ThermoFisher Scientific). qRT-PCR was performed using Power SYBR Green Master Mix (ThermoFisher Scientific) on StepOnePlus instrument (Applied Biosystems) using the housekeeping gene $r p s L$ as an internal reference for quantification. Expression of five major RND efflux pump related genes $($ oprM, mexB, mexD, mexF and mexY) were assessed using published sets of primers (SI Appendix. Table S2). After normalization to the expression of $r p s L$, the levels of five pump genes were compared under different conditions at $5 \mathrm{~h}, 9 \mathrm{~h}$ and 12 h.

Assays of efflux pumps Firstly, PA $\beta \mathrm{N}$ as a specific pump inhibitor was used to investigate the efficiency of pumps in the presence of streptomycin. Furthermore, the growth curves of $P$. aeruginosa PAO1 supplemented with $20 \mu \mathrm{g} / \mathrm{mL}$ streptomycin were measured, initially treated with $10 \mu \mathrm{mol} / \mathrm{L} \mathrm{CCCP}$ and $1 \mathrm{mmol} / \mathrm{L}$ sodium azide, respectively. The growth curves were measured by a plate reader (Tecan) at the wavelength of $600 \mathrm{~nm}$ (A600). $50 \mu \mathrm{L}$ mineral oil was added to prevent evaporation during long term culture.

Statistical analysis and software The statistical significance of differences was determined by a Student's $t$-test. The fluorescent intensities and fold changes in mRNA levels were normalized prior to analysis. GraphPad Prism 5.0 was used for analyzing the data and generating figures.

\section{ACKNOWLEDGMENTS}

We thank D. K. Newman (Caltech) for sharing PA14 and its mutants ( $\Delta$ phr, $\Delta$ soxR and $\Delta s o x$ R-phs) and purified PYO, PCA, PCN and 1-OHPHZ, H. E. Blackwell (UW-Madison) for sharing PAO-JP2, L. S. Thomashow (WSU) for sharing PAO1-W and its mutants (PAO-mxM and PAO-mxS), D. K. Newman for insightful discussions and critical reading of the earlier drafts 
of the manuscript, and B. Shao (Beijing CDC) for LC-MS/MS analysis.

\section{REFERENCES}

1. Brown D (2015) Antibiotic resistance breakers: can repurposed drugs fill the antibiotic discovery void? Nat Rev Drug Disco 14:821-832.

2. Clatworthy AE, Pierson E, Hung DT (2007) Targeting virulence: a new paradigm for antimicrobial therapy. Nat Chem Biol 3:541-548.

3. Blair JM, Webber MA, Baylay AJ, Ogbolu DO, Piddock LJ (2015) Molecular mechanisms of antibiotic resistance. Nat Rev Microbiol 13:42-51.

4. Coates AR, Halls G, Hu Y (2011) Novel classes of antibiotics or more of the same? Brit J Pharmacol 163:184-194.

5. Projan SJ (2003) Why is big Pharma getting out of antibacterial drug discovery? Curr Opin Microbiol 6:427-430.

6. Boucher HW, et al. (2009) Bad bugs, no drugs: no ESKAPE! An update from the Infectious Diseases Society of America. Clin Infect Dis 48:1-12.

7. Lopatkin AJ, et al. (2016) Antibiotics as a selective driver for conjugation dynamics. Nat Microbiol $1: 16044$.

8. Lopatkin AJ, Meredith HR, Srimani JK, Pfeiffer C, Durrett R, You L (2017) Persistence and reversal of plasmid-mediated antibiotic resistance. Nat Commun 8:1689.

9. Brauner A, Fridman O, Gefen O, Balaban NQ (2016) Distinguishing between resistance, tolerance and persistence to antibiotic treatment. Nat Rev Microbiol 14:320-330.

10. Meredith HR, Srimani JK, Lee AJ, Lopatkin AJ, You L (2015) Collective antibiotic tolerance: mechanisms, dynamics and intervention. Nat Chem Biol 11:182-188. 
11. Handwerger S, Tomasz A (1985) Antibiotic tolerance among clinical isolates of bacteria. Annu Rev Pharmacol 25:349-380.

12. Andersson DI, Hughes D (2014) Microbiological effects of sublethal levels of antibiotics. Nat Rev Microbiol 12:465-478.

13. Balaban NQ, Merrin J, Chait R, Kowalik L, Leibler S (2004). Bacterial persistence as a phenotypic switch. Science 305:1622-1625.

14. Levin-Reisman I, Ronin I, Gefen O, Braniss I, Shoresh N, Balaban NQ (2017) Antibiotic tolerance facilitates the evolution of resistance. Science 355:826-830.

15. Price-Whelan A, Dietrich LE, Newman DK (2006) Rethinking 'secondary' metabolism: physiological roles for phenazine antibiotics. Nat Chem Biol 2:71-78.

16. Folkesson A et al. (2012) Adaptation of Pseudomonas aeruginosa to the cystic fibrosis airway: an evolutionary perspective. Nat Rev Microbiol 10:841-851.

17. Dietrich LE, Price-Whelan A, Petersen A, Whiteley M, Newman DK (2006) The phenazine pyocyanin is a terminal signaling factor in the quorum sensing network of Pseudomonas aeruginosa. Mol Microbiol 61:1308-1321.

18. Ramos I, Dietrich LE, Price-Whelan A, Newman DK (2010) Phenazines affect biofilm formation by Pseudomonas aeruginosa in similar ways at various scales. Res Microbiol 161:187-191.

19. Wang Y, Newman DK (2008) Redox reactions of phenazine antibiotics with ferric (hydr)oxides and molecular oxygen. Environ Sci Technol 42:2380-2386.

20. Dietrich LE, Teal TK, Price-Whelan A, Newman DK (2008) Redox-active antibiotics control gene expression and community behavior in divergent bacteria. Science 321:1203-1206.

21. Laursen JB, Nielsen J (2004) Phenazine natural products: biosynthesis, synthetic analogues, and 
biological activity. Chem Rev 104:1663-1686.

22. Essar DW, Eberly L, Hadero A, Crawford IP (1990) Identification and characterization of genes for a second anthranilate synthase in Pseudomonas aeruginosa: interchangeability of the two anthranilate synthases and evolutionary implications. J Bacteriol 172:884-900.

23. Shen L et al. (2008) Modulation of secreted virulence factor genes by subinhibitory concentrations of antibiotics in Pseudomonas aeruginosa. J Microbiol 46:441-447.

24. Babić F, Venturi V, Maravić-Vlahovicek G (2010) Tobramycin at subinhibitory concentration inhibits the RhlI/R quorum sensing system in a Pseudomonas aeruginosa environmental isolate. BMC Infect Dis 10:148.

25. Husain FM, Ahmad I (2013) Doxycycline interferes with quorum sensing-mediated virulence factors and biofilm formation in Gram-negative bacteria. World J Microbiol Biotechnol. 29:949-957.

26. Nicastro GG et al. (2014) Cyclic-di-GMP levels affect Pseudomonas aeruginosa fitness in the presence of imipenem. Environ Microbiol 16:1321-1333.

27. Hoffman LR et al. (2005) Aminoglycoside antibiotics induce bacterial biofilm formation. Nature 436:1171-1175.

28. Malone JG et al. (2010) YfiBNR mediates cyclic di-GMP dependent small colony variant formation and persistence in Pseudomonas aeruginosa. PLoS Pathog 6:e1000804.

29. Okegbe C et al. (2017) Electron-shuttling antibiotics structure bacterial communities by modulating cellular levels of c-di-GMP. Proc Natl Acad Sci USA 114:E5236-5245.

30. Hunter RC, Klepac-Ceraj V, Lorenzi MM, Grotzinger H, Martin TR, Newman DK (2012) Phenazine content in the cystic fibrosis respiratory tract negatively correlates with lung function and microbial complexity. Am J Respir Cell Mol Biol 47:738-745. 
31. Pearson JP, Pesci EC, Iglewski BH (1997) Roles of Pseudomonas aeruginosa las and rhl quorum-sensing systems in control of elastase and rhamnolipid biosynthesis genes. J Bacteriol 179:5756-5757.

32. Mavrodi DV et al. (2001) Functional analysis of genes for biosynthesis of pyocyanin and phenazine-1-carboxamide from Pseudomonas aeruginosa PAO1. J Bacteriol 183:6454-6465.

33. Green J, Page MS (2004) Bacterial redox sensors. Nat Rev Microbiol, 2:954-966.

34. Glasser NR, Kern SE, Newman DK (2014) Phenazine redox cycling enhances anaerobic survival in Pseudomonas aeruginosa by facilitating generation of ATP and a proton-motive force. Mol Microbiol 92:399-412.

35. Winter SE et al. (2010) Gut inflammation provides a respiratory electron acceptor for Salmonella. Nature 467:426-429.

36. Winter SE et al. (2013) Host-derived nitrate boosts growth of E. coli in the inflamed gut. Science 339:708-711.

37. Shatalin K, Shatalina E, Mironov A, Nudler E (2011) $\mathrm{H}_{2} \mathrm{~S}$ : a universal defense against antibiotics in bacteria. Science 334:986-990.

38. Conrad C et al. (2015) Long-term treatment with oral $N$-acetylcysteine: affects lung function but not sputum inflammation in cystic fibrosis subjects. A phase II randomized placebo-controlled trial. J Cyst Fibros 14:219-227.

39. Lovley DR et al. (1996) Humic substances as electron acceptors for microbial respiration. Nature 382:445-448.

40. Wang Y, Kern SE, Newman DK (2010) Endogenous phenazine antibiotics promote anaerobic survival of Pseudomonas aeruginosa via extracellular electron transfer. J Bacteriol 192:365-369.

41. Kashket ER (1985) The proton motive force in bacteria: a critical assessment of methods. Annu Rev 
Microbiol 39:219-242.

42. Strahl H, Hamoen LW (2010) Membrane potential is important for bacterial cell division. Proc Natl Acad Sci USA 107:12281-12286.

43. Paulsen IT, Brown MH, Skurray RA (1996) Proton-dependent multidrug efflux systems. Microbiol Rev 60:575-608.

44. Piddock LJ (2006) Multidrug-resistance efflux pumps - not just for resistance. Nat Rev Microbiol 4:629-636.

45. Greenberg EP (2000) Bacterial genomics: Pump up the versatility. Nature 406:947-948.

46. Kiser TH, Obritsch MD, Jung R, MacLaren R, Fish DN (2010) Efflux pump contribution to multidrug resistance in clinical isolates of Pseudomonas aeruginosa. Pharmacotherapy 30:632-638.

47. Masuda N et al. (2000) Substrate specificities of MexAB-OprM, MexCD-OprJ, and MexXY-oprM efflux pumps in Pseudomonas aeruginosa. Antimicrob Agents Chemother 44:3322-3327.

48. Allison KR, Brynildsen MP, Collins JJ (2011) Metabolite-enabled eradication of bacterial persisters by aminoglycosides. Nature 473:216-220.

49. Bowler MW, Montgomery MG, Leslie AG, Walker JE (2006) How azide inhibits ATP hydrolysis by the F-ATPases. Proc Natl Acad Sci USA 103:8646-8649.

50. Poole K (2005) Efflux-mediated antimicrobial resistance. J Antimicrob Chemother 56:20-51.

51. Costa KC, Bergkessel M, Saunders S, Korlach J, Newman DK (2015) Enzymatic degradation of phenazines can generate energy and protect sensitive organisms from toxicity. MBio 6:e01520-15.

52. Belenky P et al. (2015) Bactericidal antibiotics induce toxic metabolic perturbations that lead to cellular damage. Cell Rep 13:968-980. 
bioRxiv preprint doi: https://doi.org/10.1101/453290; this version posted October 25, 2018. The copyright holder for this preprint (which was not certified by peer review) is the author/funder, who has granted bioRxiv a license to display the preprint in perpetuity. It is made available under aCC-BY 4.0 International license.

\section{Supporting Information Legends}

Material and methods

Tables S1 to S2

Figs. S1 to S10

References (1-9) 


\section{Figure legends}

Fig. 1. Antibiotics induced accumulation of PYO. (A) Kanamycin induced color changes in $P$. aeruginosa PAO1 cultured in LB medium. (B) PYO concentration and cell density (A600 nm) of PAO1 in varying concentrations of kanamycin. (C) Typical dynamics of PYO concentrations and bacterial growth curves of PAO1 treated with $20 \mu \mathrm{g} / \mathrm{mL}$ kanamycin. (D) Different classes of antibiotics promoted c-di-GMP accumulation in PAO1. Each data point was normalized with respect to the concentration of c-di-GMP in the absence of antibiotic treatment. Means \pm s.d. were presented for $\mathbf{A}$ to $\mathbf{C}(\mathrm{n}=3)$, for $\mathbf{D}(\mathrm{n}=6)$.

Fig. 2. PYO-mediated tolerance against antibiotic treatment. (A) PYO-mediated tolerance in P. aeruginosa PAO1. Various antibiotics were tested, including $50 \mu \mathrm{g} / \mathrm{mL}$ kanamycin, $4 \mu \mathrm{g} / \mathrm{mL}$ gentamicin, $20 \mu \mathrm{g} / \mathrm{mL}$ streptomycin, $20 \mu \mathrm{g} / \mathrm{mL}$ tobramycin, $0.5 \mu \mathrm{g} / \mathrm{mL}$ norfloxacin, $7.5 \mu \mathrm{g} / \mathrm{mL}$ chloramphenicol and $50 \mu \mathrm{g} / \mathrm{mL}$ carbenicillin and $1.0 \mu \mathrm{g} / \mathrm{mL}$ polymyxin B. Means \pm s.d. were presented $(\mathrm{n}=3)$. (B) PYO-mediated tolerance in Gram-negative bacteria, including P. aeruginosa PAO1, Escherichia coli (MG1655 and ESBL-expressing strain), and Salmonella Typhimurium, and Gram-positive bacteria, including Bacillus cereus, Bacillus subtilis, and Staphylococcus aureus. Bacteria were cultured in LB media supplemented with $20 \mu \mathrm{g} / \mathrm{mL}$ streptomycin, except $5 \mu \mathrm{g} / \mathrm{mL}$ streptomycin for B. cereus. Means \pm s.d. were presented $(n=6)$. $p$-values between streptomycin treated bacteria with and without PYO were less than 0.01. In addition to PAO1, PYO conferred antibiotic tolerance to several other strains of P. aeruginosa, including PA1C (ATCC 15692), PAO-JP2 with $\Delta l a s I$ and $\Delta r b l I(31), \mathrm{PAO} 1-\mathrm{W}$ and its mutants (PAO-mxM and PAO-mxS) (32), PA14 and its mutant (PA14 $\Delta p h r)(17)$. Among these, PAO-mxM, PAO-mxS and PA14 $\Delta p h z$ cannot synthesize PYO, indicating that PYO-mediated tolerance is independent of the strain's ability to synthesize it (Supplementary, Fig. S5). The tolerance was maintained for other Gram-negative (Escherichia coli, Salmonella Typhimurium) and Gram-positive (Bacillus cereus, Bacillus subtilis and Staphylococcus aureus) bacteria treated with antibiotics (Fig. 2B). 


\section{Fig. 3. PYO mediates antibiotic tolerance by enhancing the cellular metabolism. (A)} PYO-mediated tolerance depends on the oxidized form of PYO. Cultures of P. aeruginosa PAO1 treated with $20 \mu \mathrm{g} / \mathrm{mL}$ streptomycin was supplemented with $15 \mu \mathrm{mol} / \mathrm{L} \mathrm{NADH}$ and $2 \mu \mathrm{g} / \mathrm{mL}$ PYO. (B) PYO served as an electron acceptor. AQDS $(1 \mu \mathrm{g} / \mathrm{mL})$ is a compound that serves as an electron receptor. It shows similar effect as PYO in offering protection of $P$. aeruginosa PAO1 against streptomycin treatment. (C) Scheme of PYO-mediated tolerance. ATP, adenosine triphosphate; EP, efflux pump; $\mathrm{NAD}^{+}$, oxidized form of nicotinamide adenine dinucleotide; $\mathrm{NADH}$, reduced form of nicotinamide adenine dinucleotide; PMF, proton-motive force; WST-8, water-soluble tetrazolium salt. (D) PYO promoted proton-motive force (PMF). Representative membrane potential of $20 \mu \mathrm{g} / \mathrm{mL}$ streptomycin treated P. aeruginosa PAO1 in the presence of either $2 \mu \mathrm{g} / \mathrm{mL}$ PYO or $10 \mu \mathrm{mol} / \mathrm{L}$ CCCP, or both as indicated. Membrane stain $\left(\mathrm{DiOC}_{2}(3)\right)$ was simultaneously added to the cultures to final concentration of $100 \mu \mathrm{mol} / \mathrm{L}$ with streptomycin and PAO1, for long term fluorescent measurement. Fluorescence of streptomycin treated PAO1 in the presence of either PYO or CCCP, or both, was normalized to that of streptomycin treated PAO1. (E) PYO-mediated tolerance was PMF dependent. Dissipation of PMF by inhibitors such as sodium azide and CCCP could significantly decrease the tolerance.

Means \pm s.d. were presented for $\mathbf{A}$ to $\mathbf{C}$, and $\mathbf{E}(\mathrm{n}=6)$, for $\mathbf{D}(\mathrm{n}=9)$.

\section{Fig. 4. PYO enhances activity of efflux pumps to export antibiotics.}

(A) Effect of PYO on EtBr efflux. Accumulation (1) was tested in P. aeruginosa PAO1 by the simultaneous addition of ethidium bromide (EtBr), streptomycin (Strep) and PYO. Fluorescence was measured after $10 \mathrm{hrs}$. Extrusion (2) was tested in P. aeruginosa PAO1 pre-treated with streptomycin and $\mathrm{EtBr}$ for $30 \mathrm{~min}$, followed by $10 \mathrm{hrs}$ of treatment with PYO. Inhibited pumps could increase the accumulation of EtBr, while activated pumps accelerated the extrusion of EtBr. Fluorescence was normalized to that of streptomycin treated PAO1 in the presence of EtBr. Means \pm s.d. were presented throughout $(n=6)$. (B) Model of antibiotics-triggered PYO accumulation to modulate tolerance. Subinhibitory concentrations of antibiotics inhibit bacterial growth (1 and 2), while promoting PYO accumulation in P. aeruginosa (3 and 4). PYO at a low level modulates universal antibiotic tolerance for various bacterial species against antibiotics (5). 

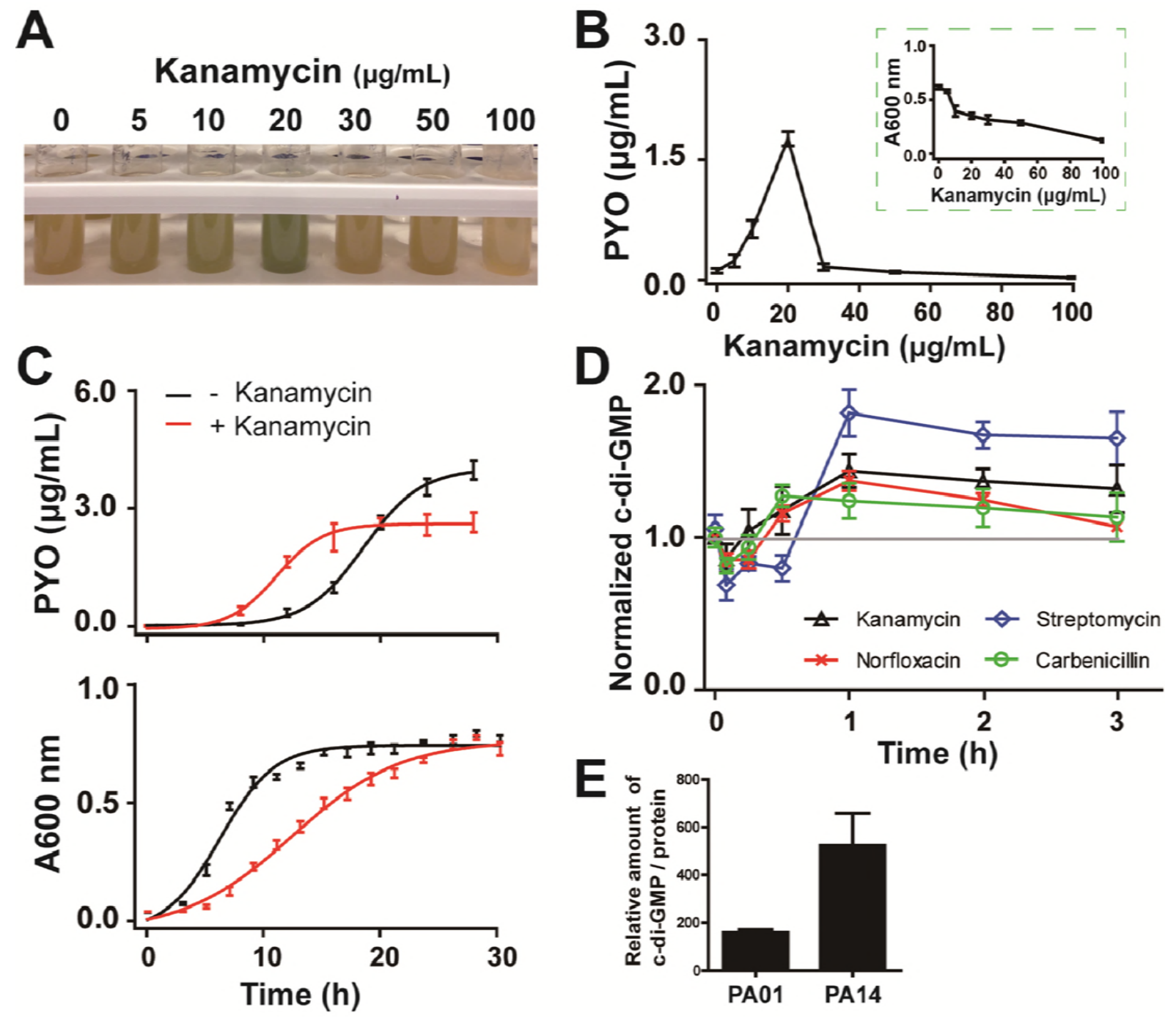

Figure 1 

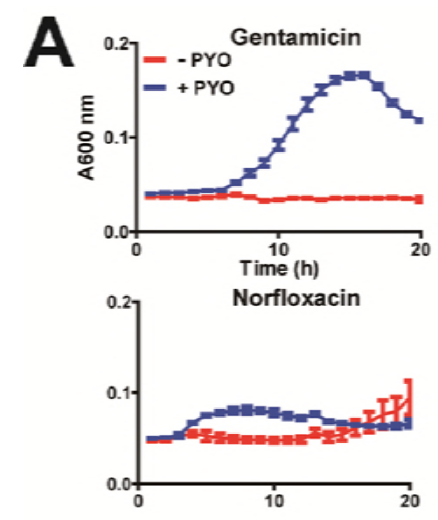
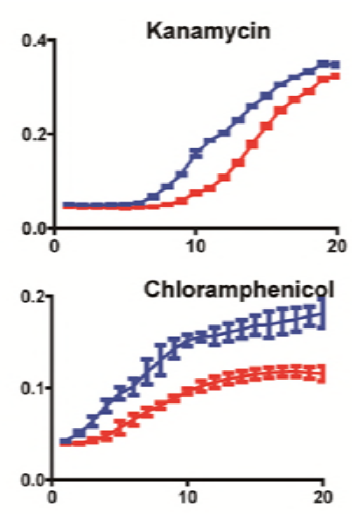
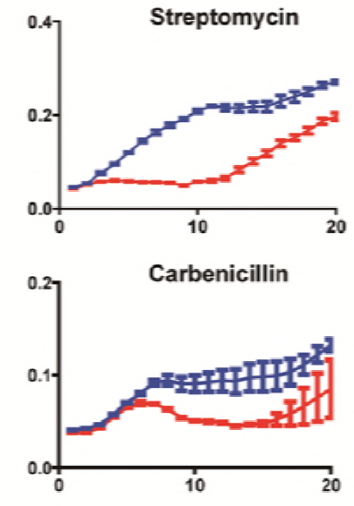
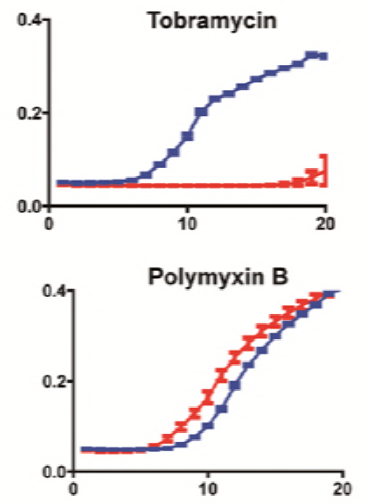

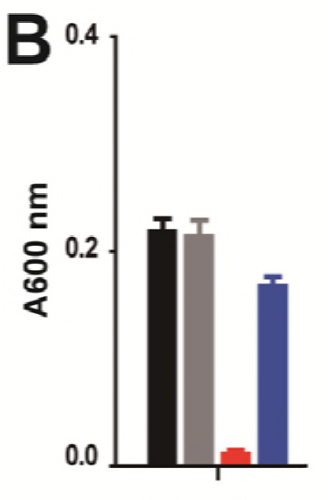

P. aeruginosa

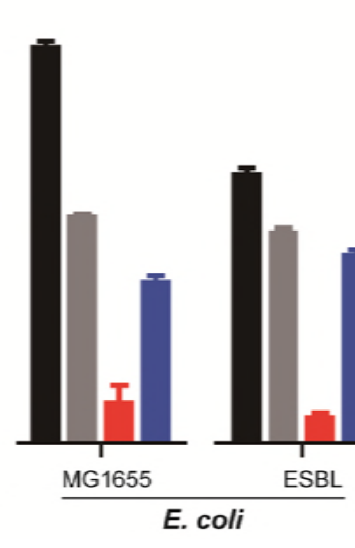

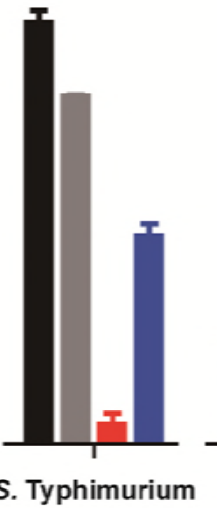

S. Typhimurium

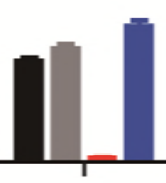

B. cereus

Bacteria

Bacteria + PYO

Bacteria + antibiotic

Bacteria + antibiotic + PYO

Figure 2 

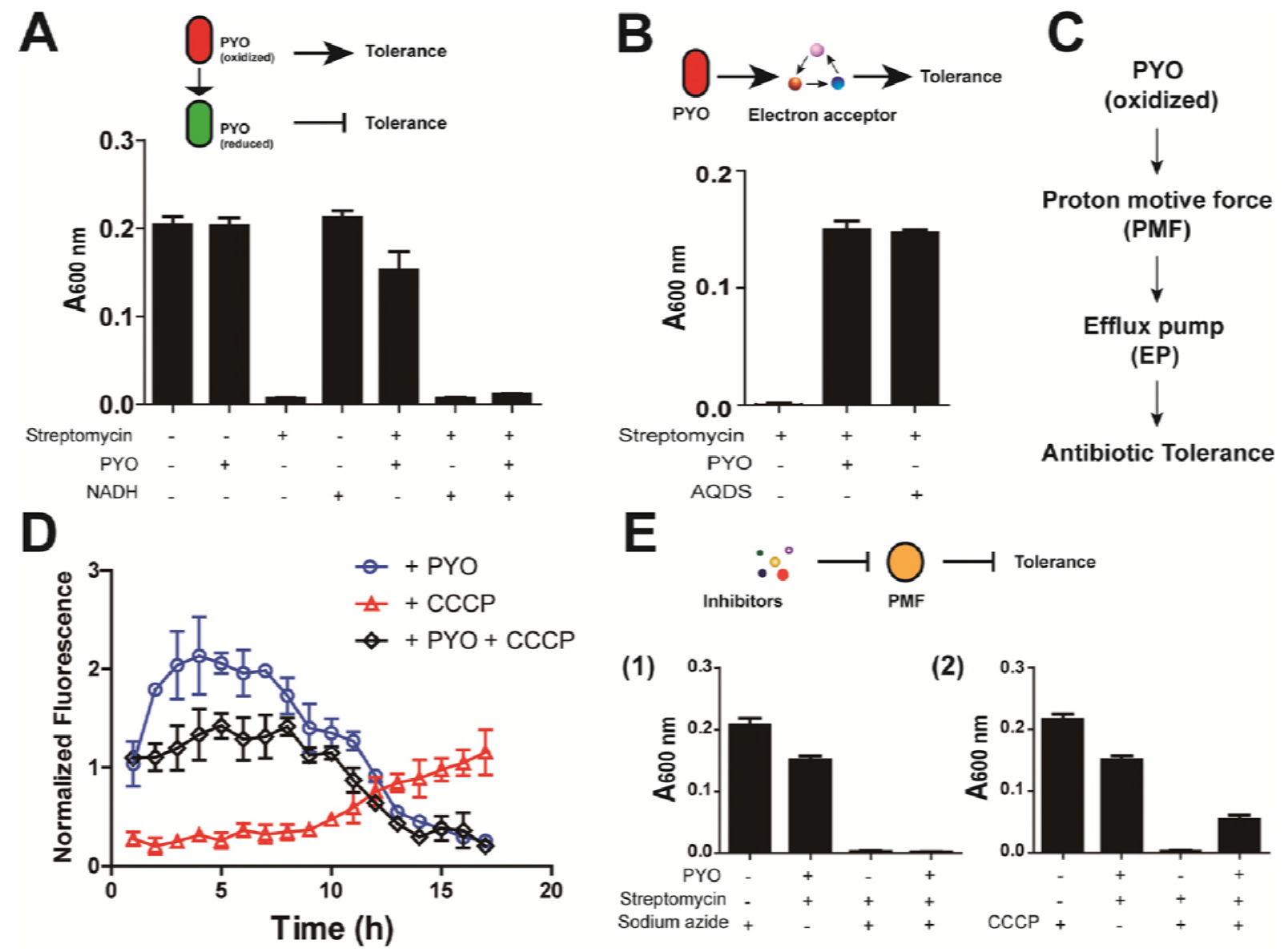

Figure 3 


\section{A (1) Accumulation}
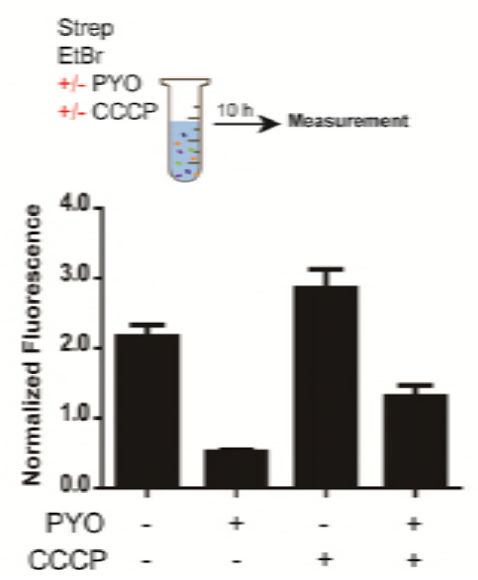

(2) Extrusion
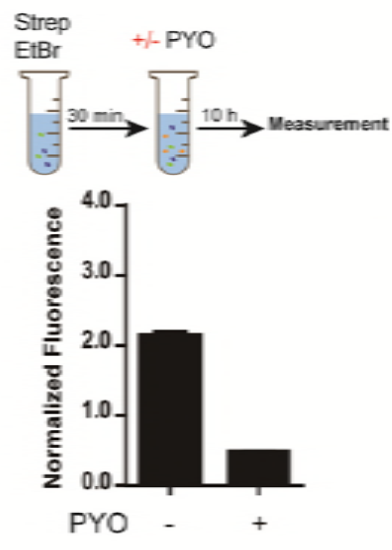

Figure 4
B

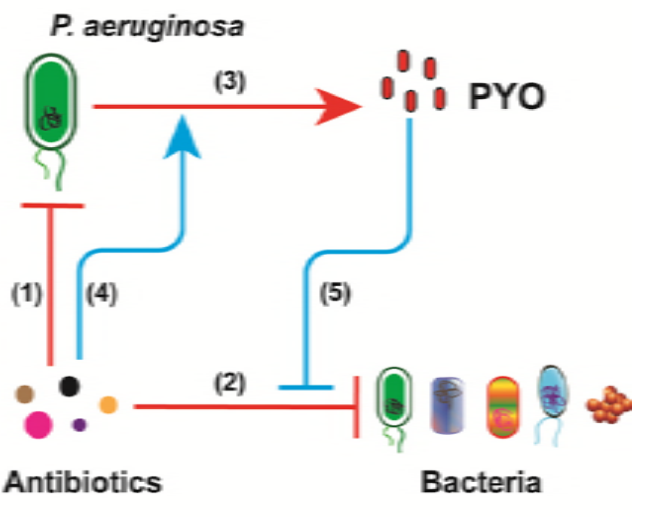

